\title{
The surface of the transneptunian object 90482 Orcus
}

\author{
C. de Bergh ${ }^{1}$, A. Delsanti ${ }^{1,2}$, G. P. Tozzi ${ }^{3}$, E. Dotto ${ }^{4}$, A. Doressoundiram ${ }^{1}$, and M. A. Barucci ${ }^{1, \star}$ \\ 1 LESIA, Observatoire de Paris, 5 place Jules Janssen, 92195 Meudon, France \\ e-mail: catherine.debergh@obspm.fr \\ 2 Institute for Astronomy, 2680 Woodlawn Drive, Honolulu, Hawaii 96822, USA \\ 3 INAF - Osservatorio Astrofisico di Arcetri, Largo E. Fermi 5, 50125 Firenze, Italy \\ 4 INAF - Osservatorio Astronomico di Roma, via Frascati 33, 00040 Monteporzio Catone (Roma), Italy
}

Received 14 December 2004 / Accepted 11 March 2005

\begin{abstract}
In April 2004, we obtained visible and near-infrared spectroscopy and photometry of the Transneptunian Object (TNO) 90482 Orcus (2004 DW) with the European Southern Observatory (ESO) 8-m telescopes of the Very Large Telescope (VLT) in Chile. This object, discovered on February 17, 2004, is one of the largest known TNOs, and it belongs to the dynamical class of Plutinos. The high signal-to-noise ratio visible spectrum recorded on April 11, 2004, is nearly flat, in good agreement with the BVRI photometry we obtained the same night and with photometric measurements from Rabinowitz et al. (2004, IAU Circ., 8295). The near-infrared spectra ( $J, H$, and $K$ bands) were obtained on April 11, 12, and 21, 2004. Relatively strong absorptions attributed to water ice were detected around 1.5 and $2 \mu \mathrm{m}$. We compare these data with spectra of Orcus obtained at the 3.56-m Telescopio Nazionale Galileo (TNG) by Fornasier et al. (2004a, A\&A, 422, L43). Only two of the TNOs observed so far exhibit stronger water ice absorptions in their spectra than Orcus. Attempts to model the entire visible-near infrared spectrum yield tentative fits with mixtures of water ice and carbonaceous compounds.
\end{abstract}

Key words. Kuiper Belt

\section{Introduction}

The study of Transneptunian objects (TNOs), which are well preserved relics from the formation of the solar sytem, is a very important new field of planetology, in which the first object was only discovered in 1992. Spectroscopy is the best method to get information on the surface composition of these objects; however, given their remoteness, small size, and low (as far as we know) albedos, this is a difficult task. There are only about 15 objects for which spectroscopy has been done so far - in particular near infrared spectroscopy, which should be the most diagnostic - while nearly 1000 objects have already been discovered. Furthermore, the studies carried out so far have revealed a general lack of diagnostic features in the spectra. Only water ice has been unambiguously identified from its near infrared signatures, and it has been seen in spectra of only a few objects (see Dotto et al. 2003; Jewitt \& Luu 2004; Licandro et al. 2005). In addition, some weak features have been seen both in the visible spectra of three Plutinos (Lazzarin et al. 2003; Fornasier et al. 2004b) and in a near infrared spectrum of the Scattered Disk TNO 26375 (1999 DE9) (Jewitt \& Luu 2001),

^ Based on observations collected during DDT time (Director Discretionary Time) at the VLT Observatory, Cerro Paranal, of the European Southern Observatory in Chile within the framework of programme 272.C-5056. which are tentatively attributed to the presence of hydrated silicates on the surface of these objects.

Plutinos are, like Pluto, objects in 3:2 mean motion resonance with Neptune. They are part of the so-called "Resonant" population of TNOs. The other two main categories of TNOs are the "Classical" TNOs and the "Scattered Disk" TNOs, which are on highly eccentric and inclined orbits. The surveys dedicated to the search of "bright" Transneptunian objects (the only ones suitable for spectroscopy) have lately led to the discovery of several such objects. One of them, 2004 DW (subsequently numbered 90482 and named Orcus), has been found recently (discovery date: February 17, 2004) using the Samuel Oschin Telescope at Palomar Mountain, California (Brown et al. 2004). Orcus, which is a Plutino, is the second brightest Transneptunian object after 90377 Sedna. Orcus is also probably one of the largest objects, although we cannot be completely sure, as its albedo has not been measured yet. Assuming an albedo lower than about 0.1 , Orcus would be larger than Charon, which has a diameter of about $1300 \mathrm{~km}$.

Visible photometric observations of Orcus were carried out at the SMARTS 1.3-m telescope at Cerro Tololo (Chile) on February 26, 2004, by Rabinowitz et al. (2004), while visible and near infrared spectroscopic observations were made at the 3.56-m TNG telescope (Canary Islands) on March 1 and 2, 2004, by Fornasier et al. (2004a). Here we present 
Table 1. The visible photometric and spectroscopic observations of 90482 Orcus.

\begin{tabular}{llllll}
\hline \hline $\begin{array}{l}\text { Date } \\
\text { (UT) }\end{array}$ & $\begin{array}{l}\text { Type of } \\
\text { measurement }\end{array}$ & $\begin{array}{l}\text { UT (start) } \\
(\mathrm{h}: \mathrm{min})\end{array}$ & $\begin{array}{l}\text { Exposure } \\
\text { time (s) }\end{array}$ & Airmass & $\begin{array}{l}\text { Measured } \\
\text { Magnitude }\end{array}$ \\
\hline April 10, 2004 & $R$ photometry & $23: 58$ & 40 & 1.097 & $R=18.85 \pm 0.03$ \\
April 10, 2004 & $V$ photometry & $23: 59$ & 60 & 1.095 & $V=19.22 \pm 0.03$ \\
April 11, 2004 & $B$ photometry & $00: 01$ & 150 & 1.094 & $B=19.90 \pm 0.03$ \\
April 11, 2004 & $I$ photometry & $00: 04$ & 60 & 1.092 & $I=18.48 \pm 0.03$ \\
April 11, 2004 & $V$ photometry & $00: 06$ & 60 & 1.089 & $V=19.23 \pm 0.03$ \\
April 11, 2004 & $V$ photometry & $00: 13$ & 60 & 1.085 & $V=19.22 \pm 0.03$ \\
\hline April 11,2004 & Spectroscopy & $00: 24$ & 600 & 1.076 & \\
April 11, 2004 & Spectroscopy & $00: 35$ & 600 & 1.075 & \\
\hline
\end{tabular}

visible and near infrared spectroscopic and photometric observations obtained in April 2004 using two different 8-m telescopes of the European Southern Observatory-Very Large Telescope (ESO-VLT) at Cerro Paranal (Chile). The spectra are of higher quality than previously obtained for this object. Photometric measurements are made for the first time in the near infrared. After discussing the data reduction and the results of our observations, we attempt to model the entire spectrum of Orcus from 0.4 to $2.4 \mu \mathrm{m}$ and make some comparisons with a few other large TNOs.

\section{Observations in the visible}

Visible observations were carried out on April 10-11, 2004, using the FORS2 (FOcal Reducer/low dispersion Spectrograph) instrument equipped with the new mosaic of two MIT $2 \mathrm{k} \times 4 \mathrm{k}, 15 \mu \mathrm{m}$ pixels, red-enhanced chips (www.eso.org/instruments/fors), and mounted at the Cassegrain focus of the Yepun (UT4) telescope of the ESO-VLT (Cerro Paranal, Chile) at the time of the observations. The observations were made nearly simultaneously with some of our infrared observations. The object was first imaged through a photometric sequence $R V B I V$ using the broadband Bessel filters $B, V$, and $I$ (central wavelengths: 429, 554, and $768 \mathrm{~nm}$, respectively), and a special $R$ filter (central wavelength: $655 \mathrm{~nm}$, FWHM: $165 \mathrm{~nm}$ ). The exposure times and UT dates are summarized in Table 1 . The $V$ filter images were repeated on purpose in order to monitor any brightness variation of the object over time. The telescope tracked at the sidereal rate, as the trailing induced by the object's apparent motion (slower than $4^{\prime \prime} / \mathrm{h}$ ) is far below the seeing value for exposure times lower than $900 \mathrm{~s}$. In addition to providing information about the object's visible colors, this photometric sequence was used as a means to identify the moving object, in order to position the slit for visible spectroscopy. Then, two consecutive spectra of $600 \mathrm{~s}$ each were acquired. We used the grism 150-I without order separation to simultaneously cover a very broad spectral range. The signal-to-noise ratio below $400 \mathrm{~nm}$ was very low and, since we used the spectrum only up to $800 \mathrm{~nm}$, there is no problem with second order overlapping. We used a $1^{\prime \prime}$ wide slit oriented in the $\mathrm{N}-\mathrm{S}$ direction. The telescope was programmed to track the apparent motion of Orcus, which was obtained from JPL ephemeris. The spectral resolution is between 100 and 200, depending on the wavelength. We also obtained, during the same night, spectra of one solar analog, SA102-1081, at two different air-masses.

\subsection{Imaging data reduction and photometry}

The images were corrected for bias and flat-field using the MIDAS software and the calibration images provided by ESO. Then, the object was measured through different apertures with radius varying from 1 time to 5 times the $F W H M$ of the Point Spread Function. The stabilization of the flux values obtained with increasing apertures was then monitored and doublechecked by the construction of a complete curve of growth (aperture radii varying from 1 to 50 pixels, i.e. $0.4^{\prime \prime}$ to $20^{\prime \prime}$ ). As the object is really bright, we did not perform any aperture correction. We then used zeropoints derived from observations of Landolt stars and provided in the framework of the ESO calibration plan, as well as typical Paranal values for atmospheric extinction and color correction to compute the final magnitudes presented in Table 1. The averaged $V$ magnitude (three measurements over a 14 min interval) is: $19.22 \pm 0.03$.

These magnitudes correspond to $B-V=0.68 \pm 0.04$, $V-R=0.37 \pm 0.04$, and $V-I=0.74 \pm 0.04$. Assuming for solar colors $B-V=0.67, V-R=0.36$, and $V-I=0.69$ (Hardorp 1980; Hartmann et al. 1990), we find that the object has nearly solar colors. This was also found by Rabinovitz et al. (2004), who made $B V R I$ photometric measurements on February 26, 2004.

Rabinowitz et al. obtained $B=19.70 \pm 0.01, V=19.01 \pm$ $0.03, R=18.64 \pm 0.02$, and $I=18.25 \pm 0.04$. Their magnitudes are therefore slightly lower than measured here. Part of it must be due to differences in geocentric $(\Delta)$ and heliocentric $(r)$ distances between February $26(\Delta=46.685$ AU, $r=47.609$ AU) and April $11(\Delta=47.094 \mathrm{AU}, r=47.616 \mathrm{AU})$. Slight differences in solar phase angles $\left(0.43^{\circ}\right.$ on February 26 , and $1^{\circ}$ on April 11) must also be taken into account. After correcting for these two effects (we used $\beta=0.2 \mathrm{mag} /$ degree for the phase angle correction; Rabinowitz, private communication), we find that, based on the measurements of Rabinovitz et al., the apparent $V$ magnitude measured on April 11 should have been 19.09 instead of 19.22. Our results are therefore very consistent with theirs. The very slight remaining difference could be due to the fact that different parts of the object were observed. Indeed, 
Table 2. The near infrared photometric and spectroscopic observations of 90482 Orcus.

\begin{tabular}{llllll}
\hline \hline $\begin{array}{l}\text { Date } \\
\text { (UT) }\end{array}$ & $\begin{array}{l}\text { Type of } \\
\text { measurement }\end{array}$ & $\begin{array}{l}\text { UT (start) } \\
\text { (h:min) }\end{array}$ & $\begin{array}{l}\text { Exposure } \\
\text { time (s) }\end{array}$ & Airmass & $\begin{array}{l}\text { Measured } \\
\text { Magnitude }\end{array}$ \\
\hline Apr. 11, 2004 & $J$ photometry & $00: 40$ & 60 & 1.07 & $J=18.19 \pm 0.03$ \\
Apr. 11, 2004 & $J$ photometry & $01: 08$ & 60 & 1.07 & $J=18.18 \pm 0.03$ \\
Apr. 11, 2004 & $H$ photometry & $01: 12$ & 84 & 1.08 & $H=17.98 \pm 0.03$ \\
Apr. 11, 2004 & $K$ s photometry & $01: 20$ & 300 & 1.08 & $K=17.98 \pm 0.03$ \\
\hline Apr. 11, 2004 & $J$ Spectroscopy & $01: 37$ & 2400 & $1.10-1.17$ & \\
\hline Apr. 11, 2004 & $J$ photometry & $02: 25$ & 60 & 1.17 & $J=18.14 \pm 0.03$ \\
Apr. 11, 2004 & $J$ photometry & $02: 40$ & 60 & 1.21 & $J=18.11 \pm 0.03$ \\
Apr. 11, 2004 & $H$ photometry & $02: 45$ & 64 & 1.22 & $H=17.99 \pm 0.03$ \\
Apr. 11, 2004 & $K$ s photometry & $02: 53$ & 300 & 1.24 & $K=17.99 \pm 0.03$ \\
\hline Apr. 12, 2004 & $J$ photometry & $01: 44$ & 60 & 1.11 & $J=18.16 \pm 0.03$ \\
Apr. 12, 2004 & $J$ photometry & $02: 18$ & 60 & 1.17 & $J=18.13 \pm 0.03$ \\
Apr. 12, 2004 & $H$ photometry & $02: 23$ & 64 & 1.18 & $H=18.06 \pm 0.03$ \\
Apr. 12, 2004 & $K$ s photometry & $02: 30$ & 300 & 1.19 & $K=17.94 \pm 0.03$ \\
\hline Apr. 12, 2004 & $H$ Spectroscopy & $02: 52$ & 3600 & $1.27-1.59$ & \\
\hline Apr. 21, 2004 & $J$ photometry & $01: 03$ & 60 & 1.10 & $J=18.10 \pm 0.03$ \\
Apr. 21, 2004 & $J$ photometry & $01: 22$ & 60 & 1.13 & $J=18.12 \pm 0.03$ \\
Apr. 21, 2004 & $H$ photometry & $01: 26$ & 64 & 1.14 & $H=18.01 \pm 0.03$ \\
Apr. 21, 2004 & $K$ s photometry & $01: 33$ & 300 & 1.15 & $K=17.97 \pm 0.03$ \\
\hline Apr. 21, 2004 & $K$ s Spectroscopy & $01: 55$ & 3600 & $1.20-1.46$ & \\
Apr. 21, 2004 & $K$ s Spectroscopy & $03: 16$ & 1200 & $1.57-1.74$ & \\
\hline
\end{tabular}

according to Ortiz (private communication), the object seems to have a lightcurve with an amplitude on the order of 0.09 mag. The corresponding absolute magnitude is $H \mathrm{v}=2.27 \pm 0.03$.

\subsection{Spectroscopic data reduction}

We made the bias, flat-field correction and wavelength calibration using MIDAS (Grosbol 1989) and the context LONG that includes routines especially developed for visible spectroscopy. The 2D spectra were sky-corrected before extraction. The two 1D spectra of 2004 DW were co-added and then divided by the $1 \mathrm{D}$ solar analog spectrum that is closest in airmass to create the final visible spectrum. Figure 1 shows the spectral reflectances (normalized to 1 at $0.55 \mu \mathrm{m}$ ) on which are plotted results from our photometric measurements. The reflectance spectrum is nearly flat, which is in good agreement with the nearly solar colors derived from the photometric measurements (see above). There is a very slight red slope, as also found by Fornasier et al. (2004a) from lower $S / N$ ratio spectra, with, maybe, the beginning of an absorption at the long wavelength end of the spectrum. No absorptions are detected at shorter wavelengths.

\section{Observations in the near infrared}

The near infrared observations were made on April 11, 12, and 21, 2004, with the Antu (UT1) telescope of ESO-VLT equipped with ISAAC (Infrared Spectrometer Array And Camera) in its SW mode (1 to $2.5 \mu \mathrm{m}$ spectral range) as a focal plane instrument (www.eso.org/instruments/isaac). Table 2 gives the circumstances of the photometric and spectroscopic observations. Broad-band images in $J, H, K$ s (central wavelengths: $1.25,1.65$, and $2.16 \mu \mathrm{m}$, respectively) were obtained in jitter mode, i.e. by moving the telescope to random positions some tens of arcsec apart. Six, eight, or ten positions (in $J, H$, and $K \mathrm{~s}$, respectively) were used, and, for each position, the typical exposure time was 10-30 s. Each night, photometric standards, sky flat fields, and dark images were also obtained. The nights of April 11 and 12 were photometric. There were thin cirrus on April 21.

The spectra in the near infrared ( $J, H$, and $K$ bands) were recorded with ISAAC in its Low Resolution spectroscopic mode. Spectra of the solar analog SA102-1081 were obtained in these bands during the same nights. The seeing was good, $0.4-0.6^{\prime \prime}$, for all the nights.

As is common in near infrared observations, the spectra were recorded in pairs, dithering the image of the object along the slit at two different positions, to reduce the contamination due to sky emissions. A typical exposure time for each exposure was $120 \mathrm{~s}$ and the slit used was $1^{\prime \prime}$ wide. The exposures were then repeated several times, up to the total exposure times indicated in Table 2 .

\subsection{Imaging data reduction and photometry}

The broad-band images were reduced by using both the automatic software Eclipse developed at ESO for ISAAC data reduction (Devillard 2001) and MIDAS with procedures especially developed for near infrared data reduction. The results 
Table 3. Averaged $V, J, H$, and $K$ s magnitudes of 90482 Orcus.

\begin{tabular}{|c|c|c|c|}
\hline $\begin{array}{l}V \\
\text { (April } 11 \text { average) }\end{array}$ & $\begin{array}{l}J \\
\text { (3 nights average) }\end{array}$ & $\begin{array}{l}H \\
\text { (3 nights average) }\end{array}$ & $\begin{array}{l}\mathrm{s} \\
\text { (3 nights average) }\end{array}$ \\
\hline $19.22 \pm 0.03$ & $18.14 \pm 0.03$ & $18.01 \pm 0.04$ & $17.97 \pm 0.03$ \\
\hline
\end{tabular}

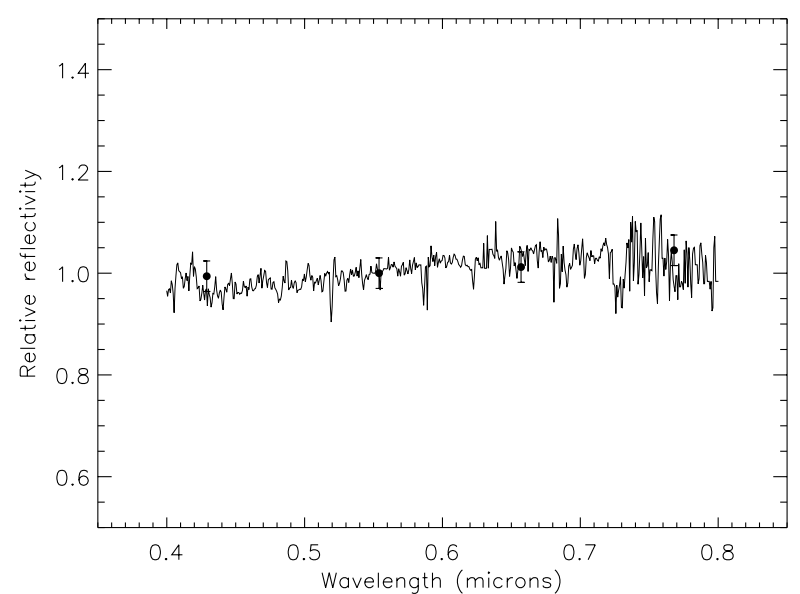

Fig. 1. The visible reflectance spectrum of 90482 Orcus normalized to 1 at $0.55 \mu \mathrm{m}$. Results of photometric measurements made the same night, and transformed into relative reflectances, are also plotted.

in these cases are identical and given in Table 2. Since the object was fairly bright, the sky background was not an important source of error. The standard deviations of all the measurements in each band were on the order of 0.03-0.04 mag. The individual magnitudes did not change significantly during a given night or from night to night. The magnitudes of Orcus averaged over the three nights of observation are reported in Table 3.

\subsection{Spectroscopic data reduction}

The spectra in the near infrared were also reduced using Eclipse and MIDAS (with procedures especially developed for spectra) software. For each spectral range $(J, H$, and $K)$, the multiple spectra recorded at each slit position $(A$ and $B)$ were averaged, and the average spectra subtracted from each other. The $(A-B)$ and $(B-A)$ 2D spectra were flat-fielded, corrected for spatial distortion, and combined, taking the offset into account. This procedure allows double elimination of the sky lines which perfectly subtract out. The 1D spectra were then extracted by integrating over the $3 \times F W H M$ of the spectrum in the slit direction, and the relative reflectivities of the TNO in each spectral band were obtained by dividing the TNO spectra by the solar analog ones. Finally, to reduce the noise, the oversampled spectra were rebinned to $50 \AA$ (spectral resolution between 240 and 500).

\section{Combined spectrum and modelling}

The infrared reflectivities were combined with the visible reflectivities using our measured $V-J, V-H$, and $V-K$ colors, derived from the averaged $V, J, H$, and $K$ s magnitudes listed in Table 3, and assuming, for solar colors, $V-J=1.07$,

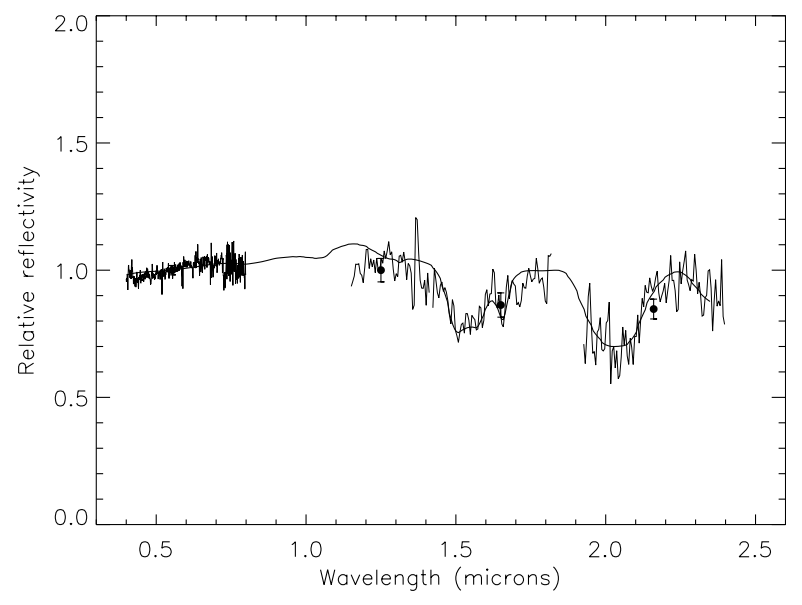

Fig. 2. The final complete reflectance spectrum of 90482 Orcus, normalized to 1 at $0.55 \mu \mathrm{m}$, with our best fit model. This model is a spatial mixture of: $5 \%$ water ice at $40 \mathrm{~K}(10 \mu \mathrm{m}), 42 \%$ kerogen $(10 \mu \mathrm{m})$, and $53 \%$ amorphous carbon $(10 \mu \mathrm{m})$. The corresponding albedo is: 0.073 at $0.55 \mu \mathrm{m}$.

$V-H=1.36, V-K=1.42$ (Hartmann et al. 1982). For the infrared part, we used the measured infrared magnitudes and the transmittance curves of the broad-band filters to place the spectra in each band. The $V$ and $J$ spectra were obtained nearly simultaneously on April 11, while the $H$ and $K$ spectra were recorded during different nights (see Table 2). Since we do not yet know the period of rotation of the object, we cannot check if we observed different parts of the object with possibly different surface properties when we obtained the $H$ and $K$ spectra. However, our approach is supported by the fact that our measured infrared magnitudes are roughly constant from night to night and, additionally, by the small lightcurve amplitude measured in the visible (Ortiz, private communication). The reflectivites were normalized to 1 at $0.55 \mu \mathrm{m}$. The final spectrum of Orcus between 0.4 and $2.4 \mu \mathrm{m}$ is shown in Fig. 2 .

The most obvious features in the near-infrared part of the spectrum are deep absorptions at 1.5 and $2 \mu \mathrm{m}$, as already found by Fornasier et al. (2004a), which are most probably due to water ice, and, maybe, the beginning of an absorption at wavelengths shorter than $1.2 \mu \mathrm{m}$. Although our near-infrared spectrum does not cover wavelengths shorter than $1.1 \mu \mathrm{m}$, in constrast to that of Fornasier et al., it is of higher quality (higher $S / N$ ratio and higher spectral resolution), and the water ice absorptions are better defined. The $1.5 \mu \mathrm{m}$ band is about $24 \%$ deep, while the $2 \mu \mathrm{m}$ band has a depth of about $30 \%$ relative to the local continuum.

In order to get more information on the nature of the surface, we made some attempts to model the visible-near infrared spectrum of Orcus. We limited ourselves to models that lead to relatively low values of the surface albedo, 


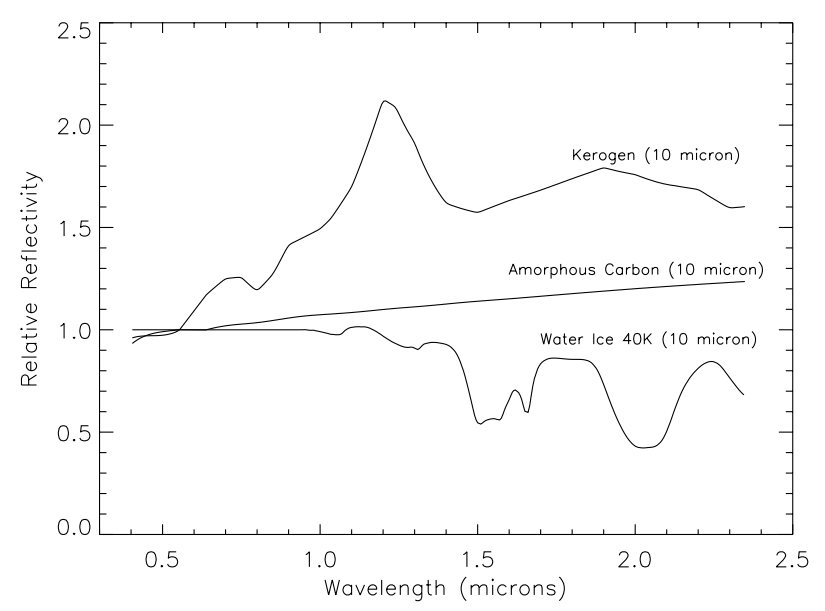

Fig. 3. Contributions of the various components used to produce the best fit model presented in Fig. 2.

as $V$ geometric albedos measured so far for TNOs range between 0.04 and 0.20 (Altenhoff et al. 2004; Cruikshank et al. 2005). A surface covered only with pure water ice is excluded; water ice must clearly be mixed with one or more components. Different types of mixtures have been considered for icy regoliths: spatial (or areal) mixtures, intimate mixtures, and intramixtures (Shkuratov et al. 1999). We chose here the simplest model, the spatial mixture, where the reflectance spectrum is a linear weighted sum of the individual component spectra, and then adopted Hapke (Hapke 1993) or Hapke-type (Douté $\&$ Schmitt 1998) radiative transfer theories to produce spectra for different grain sizes from optical constants, when available.

For water ice, we used the optical constants of crystalline water ice at expected TNOs surface temperatures (40 to $60 \mathrm{~K}$ ) measured by Grundy \& Schmitt (1998) between 0.8 and $2.5 \mu \mathrm{m}$ and, for lack of more appropriate data, those of water ice at $270 \mathrm{~K}$ from Warren (1984) between 0.4 and $0.8 \mu \mathrm{m}$. We found that an areal coverage of only a few percent of pure water ice was sufficient to account for the depths of the absorptions, but that it was necessary to add one or more components with a moderate "red" slope in the infrared. Plausible constituents with red slopes in the infrared include silicates and carbonaceous compounds, such as some types of amorphous carbon, which is a very dark material, tholins and kerogens. None of the silicates we tried provided a good fit, as they produced very broad absorptions at 1 and $2 \mu \mathrm{m}$ which are not observed. For the carbonaceous compounds included in our modelling attempts, we used amorphous carbon as measured by Zubko et al. (1996), Titan tholins from Khare et al. (1984), and kerogens from Clark et al. (1993) and Khare et al. (1991) (type II kerogen).

The best fit was obtained when two types of carbonaceous compounds were used: amorphous carbon and kerogen. Our best fit model (Fig. 2) includes: $5 \%$ of water ice at $40 \mathrm{~K}$ (grain size: $10 \mu \mathrm{m}), 42 \%$ of type II kerogens (grain size: $10 \mu \mathrm{m}$ ), and $53 \%$ of amorphous carbon (grain size: $10 \mu \mathrm{m}$ ). The corresponding geometric albedo at $0.55 \mu \mathrm{m}$ is 0.073 , which is within the limits of albedos measured so far for TNOs. Figure 3 shows separately the contributions of the various components used to produce our best fit model. But this model is certainly not unique. The quality of the data, the limited number of diagnostic features detected, and, most of all, the lack of constraints on the surface albedo are major limitations that prevent us from going any further at this point.

Although tentative, our model indicates unambiguously that the surface of Orcus is covered by some percentage of water ice, in good agreement with the work of Fornasier et al. (2004a). The water ice could be distributed as patches of relatively clean ice or as contaminated water ice over a much larger part or even the whole surface of the TNO, or a combination of both. Although TNOs are expected to be made up in large part of water ice, very few of the objects studied so far present strong water ice absorptions in their spectra. It is postulated that for most TNOs, the water ice at the surface has been covered by other materials or sufficiently contaminated and irradiated so that it is no longer detectable. The presence of very apparent water ice on a TNO may indicate a young surface, either because of a relatively recent collision that has revealed unexposed material from the interior, or because of cometary-type activity (or cryovolcanism; see Jewitt \& Luu 2004). In the next section we consider TNOs which present spectral characteristics somewhat similar to those of Orcus, in order to see if they have other common characteristics, orbital or physical.

\section{Discussion}

The main characteristics of the reflectance spectrum of Orcus are not only relatively strong absorptions of water ice in the near infrared but also a nearly flat spectrum in the visible. Only three Transneptunians among those observed so far (Pluto and Charon included) have spectra with similar characteristics: $19308\left(1996 \mathrm{TO}_{66}\right), 55636\left(2002 \mathrm{TX}_{300}\right)$, and Charon. The first two have very strong water ice absorptions in their spectra (Brown et al. 1999; Licandro et al. 2005), although in the case of $19308\left(1996 \mathrm{TO}_{66}\right)$, the very strong absorptions are seen on only part of the object. They are both Classical TNOs, and curiously, have very similar orbital characteristics (see Table 4). Cometary activity has been suspected for 19308 (1996 TO 66 ), based on observed variations in its lightcurve (see Table 4). Another Classical TNO, 50000 Quaoar, also has significant water ice absorptions in its spectrum, albeit more subdued than for the three objects listed above (Jewitt \& Luu 2004); but it is a very red object in the visible (Fornasier et al. 2004b). Its nearly circular orbit has low inclination.

The spectrum of Orcus resembles more closely that of Charon, which has also a nearly flat spectrum in the visible (Fink \& DiSanti 1988) and weaker water ice absorptions than $19308\left(1996 \mathrm{TO}_{66}\right)$ and $55636\left(2002 \mathrm{TX}_{300}\right)$, as summarized in Table 4. For Charon spectra in the near infrared, see, e.g., Brown \& Calvin (2000); Dumas et al. (2001). The presence of a feature at $1.65 \mu \mathrm{m}$ in the spectrum of Charon indicates unambiguously that water ice at the surface of Pluto's satellite is in the crystalline state. Our spectrum of Orcus is not of sufficient quality to determine with certainty the state of the ice from the presence or absence of the $1.65 \mu \mathrm{m}$ feature typical of cold crystalline water ice, but the relatively good fit achieved in the $1.5 \mu \mathrm{m}$ band favors crystalline ice, as opposed to amorphous ice; see Schmitt et al. (1998) for a comparison between spectra of amorphous and crystalline ice. Likewise, we do not 
Table 4. Some characteristics of TNOs with neutral visible colors and strong water ice absorptions in their spectra.

\begin{tabular}{|c|c|c|c|c|}
\hline Object & $\begin{array}{l}\text { TNO } 19308 \\
\left(1996 \text { TO }_{66}\right)\end{array}$ & $\begin{array}{l}\text { TNO } 55636 \\
\left(2002 \mathrm{TX}_{300}\right)\end{array}$ & Charon & $\begin{array}{l}\text { TNO } 90482 \\
\text { Orcus }\end{array}$ \\
\hline Perihelion & 37.96 AU & $37.88 \mathrm{AU}$ & $29.69 \mathrm{AU}$ & $30.872 \mathrm{AU}$ \\
\hline Aphelion & 48.338 AU & $48.32 \mathrm{AU}$ & 49.85 AU & $48.075 \mathrm{AU}$ \\
\hline Eccentricity & 0.120 & 0.121 & 0.25 & 0.218 \\
\hline Inclination & $27.5^{\circ}$ & $25.9^{\circ}$ & $17.13^{\circ}$ & $20.6^{\circ}$ \\
\hline$H v$ magnitude & 4.5 & 3.3 & 1. & $2.27^{a}$ \\
\hline Light-curve amplitude & variable ${ }^{b}$ & $0.09 \pm 0.08^{c}$ & $0.08^{d}$ & about $0.09^{e}$ \\
\hline Visible geometric albedo & $?$ & $\geq 0.084^{c}$ & $0.36-0.39^{d}$ & $?$ \\
\hline $2-\mu \mathrm{m}$ band depth & $70 \%$ & $80 \%$ & $60 \%$ & $30 \%$ \\
\hline $1.5-\mu \mathrm{m}$ band depth & $50 \%$ & $66 \%$ & $42 \%$ & $24 \%$ \\
\hline
\end{tabular}

${ }^{a}$ This work, ${ }^{b} 0.12$ (double-peak) in 1997, 0.33 (single-peak) in 1998 (Hainaut et al. 2000), ${ }^{c}$ Ortiz et al. (2004), ${ }^{d}$ Buie et al. (1997), ${ }^{e}$ Ortiz, private communication.

have enough $S / N$ ratio to determine if the weak absorption detected at $2.2 \mu \mathrm{m}$ (and tentatively assigned to some ammonia compound; Brown \& Calvin 2000) in some spectra of Charon is also present.

Orcus is a Plutino, which means that it is in 3:2 resonance with Neptune, as is the Pluto-Charon binary system. Its orbital eccentricity and inclination are quite similar to those of the Pluto-Charon binary (see Table 4). However, Charon's visible geometric albedo is $0.36-0.39$, which is higher than the albedos measured so far for TNOs. But higher albedos for large TNOs with strong water absorptions in their spectra cannot be excluded. The albedo measurements currently carried out with the Spitzer Space Telescope are essential for a meaningful comparison among these four objects, and to determine if Charon is indeed peculiar because of its proximity to the "big" Pluto or if it resembles some other large TNOs. Higher quality near infrared spectra would also be very helpful.

Acknowledgements. We are very grateful to J.-L. Ortiz, J. Licandro, and D. Rabinowitz for communicating their results to us in advance of publication, to B. Schmitt for providing us with the spectra of water ice, and to D. Strobel for a careful reading of the paper.

\section{References}

Altenhoff, W. J., Bertoldi, F., \& Menten, K. M. 2004, A\&A, 415, 771 Brown, M. E., \& Calvin, W. M. 2000, Science, 287, 107

Brown, R. H., Cruikshank, D. P., \& Pendleton, Y. J. 1999, ApJ, 519, L101

Brown, M. E., Rabinowitz, D. L., \& Trujillo, C. A. 2004, IAU Circ., 8291

Buie, M. W., Tholen, D. J., \& Wasserman, L. H. 1997, Icarus, 125, 233

Clark, R. N., Swayze, G. A., Gallagher, A. J., King, T. V. V., \& Calvin, W. M. 1993, US Geological Survey Open File Report 93-592, http://speclab.cr.usgs.gov

Cruikshank, D. P., Stansberry, J. A., Emery, J. P., et al. 2005, submitted

Devillard, N. 2001, in Astronomical Data Analysis Software and Systems X, ed. F. R. Harnden, Jr., F. A. Primini, \& H. E. Payne (San Francisco: ASP), ASP Conf. Ser., 238, 525
Dotto, E., Barucci, M. A., \& de Bergh, C. 2003, Comptes Rendus Physique, 4, 775

Douté, S., \& Schmitt, B. 1998, J. Geophys. Res., E103, 31,367

Dumas, C., Terrile, R. J., Brown, R. H., Schneider, G., \& Smith, B. A. 2001, AJ, 121, 1163

Fink, U., \& DiSanti, M. A. 1988, AJ, 95, 229

Fornasier, S., Dotto, E., Barucci, M. A., \& Barbieri, C. 2004a, A\&A, 422, L43

Fornasier, S., Doressoundiram, A., Tozzi, G. P., et al. 2004b, A\&A, 421, 353

Grosbol, P. 1989, Rev. Modern Astron., 2, 242

Grundy, W. M., \& Schmitt, B. 1998, J. Geophys. Res., E103, 25,809

Hainaut, O. R., Delahodde, C. E., Boehnhardt, H., et al. 2000, A\&A, 356,1076

Hapke 1993, Theory of Reflectance and Emittance Spectroscopy (New-York: Cambridge Univ. Press)

Hardorp, J. 1980, A\&A, 91, 221

Hartmann, W. K., Cruikshank, D. P., \& Degewij, J. 1982, Icarus, 52, 377

Hartmann, W. K., Tholen, D., Meech, K., \& Cruikshank, D. 1990, Icarus, 83,1

Jewitt, D. C., \& Luu, J. 2001, AJ, 122, 2099

Jewitt, D. C., \& Luu, J. 2004, Nature, 432, 731

Khare, B. N., Sagan, C., Arakawa, E. T., et al. 1984, Icarus, 60, 127

Khare, B. N., Thompson, W. R., Sagan, C., et al. 1991, in Origin and Evolution of Interplanetary Dust, IAU Colloq. 126, ed. A. C. Levasseur-Regourd, \& H. Hasegawa (Kluwer Academic Publishers), ASSL, 173, 99

Lazzarin, M., Barucci, M. A., Boehnhardt, H., et al. 2003, AJ, 125, 1554

Licandro, J., di Fabrizio, L., Pinilla-Alonso, N., et al. 2005, submitted

Ortiz, J. L., Sota, A., Moreno, R., et al. 2004, A\&A, 420, 383

Rabinowitz, D., Tourtellotte, S., Brown, M. E., \& Trujillo, C. A. 2004, IAU Circ., 8295

Schmitt, B., Quirico, E., Trotta, F., \& Grundy, W. M. 1998, in Solar System Ices, ed. B. Schmitt, C. de Bergh, \& M. Festou (Kluwer Academic Publishers), 199

Shkuratov, Y., Starukhina, L., Hoffmann, H., \& Arnold, G. 1999, Icarus, 137, 235

Warren, S. G. 1984, Appl. Opt., 23, 1206

Zubko, V. G., Mennella, V., Colangeli, L., \& Bussoletti, E. 1996, MNRAS, 282, 1321 